\title{
CRP/prealbumin, a novel inflammatory index for predicting recurrence after radical resection in gastric cancer patients: post hoc analysis of a randomized phase III trial
}

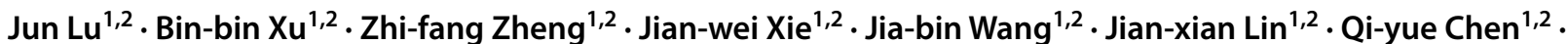 \\ Long-long $\mathrm{CaO}^{1,2} \cdot \mathrm{Mi} \mathrm{Lin}^{1,2} \cdot \mathrm{Ru}$-hong $\mathrm{Tu}^{1,2} \cdot$ Ze-ning Huang ${ }^{1,2}$. Chao-hui Zheng ${ }^{1,2}$. Chang-Ming Huang ${ }^{1,2}$. \\ Ping $\mathrm{Li}^{1,2}$
}

Received: 31 July 2018 / Accepted: 25 October 2018 / Published online: 30 October 2018

(c) The International Gastric Cancer Association and The Japanese Gastric Cancer Association 2018

\begin{abstract}
Background Serum prealbumin (PALB) can predict the prognosis of patients with gastric cancer (GC). However, the prognostic value of combination of C-reactive protein and PALB (CRP/PALB) remains unclear.

Methods A total of 419 gastric cancer patients included in a clinical trial (NCT02327481) were analyzed. The present study is a substudy of the trial. Receiver operating characteristic (ROC) curves were generated, and by calculating the areas under the curve (AUC) and the C-index, the discriminative ability of each inflammatory index was compared, including CRP/ PALB, C-reactive protein/albumin, Glasgow prognostic score (GPS), modified GPS, systemic immune-inflammation index, neutrophil-lymphocyte ratio, and platelet-lymphocyte ratio.

Results Ultimately, 401 patients were included in this study. The optimal cutoff value of CRP/PALB was 17.7. According to this cutoff point, the entire sample was divided into a CRP/PALB $<17.7$ (LCP) group and a CRP/PALB $\geq 17.7$ (HCP) group, comprising 245 and 156 patients, respectively. There were 54 and 22 patients experienced recurrence in the HCP and LCP group, respectively, $p<0.001$. Compared with traditional inflammatory indices, CRP/PALB had the highest AUC (0.707) and C-index (0.716), all $p<0.05$. The post-recurrence survival (PRS) of patients in the HCP group was significantly shorter than that in the LCP group $(p=0.010)$, especially for pathological stage III patients $(p=0.015)$ or patients with distant $(p=0.018)$ or local $(p=0.023)$ recurrences.

Conclusions The predictive value of preoperative CRP/PALB for the recurrence of GC is significantly better than traditional inflammatory indices. HCP significantly reduces the PRS, especially for pathological stage III patients or patients with distant or local recurrences.
\end{abstract}

Keywords Gastric cancer · Inflammatory index $\cdot \mathrm{CRP} /$ prealbumin $\cdot$ Recurrence $\cdot$ Post-recurrence survival

Jun Lu and Bin-bin Xu contributed equally to this work and should be considered co-first authors.

Electronic supplementary material The online version of this article (https://doi.org/10.1007/s10120-018-0892-0) contains supplementary material, which is available to authorized users.

Chao-hui Zheng wwkzch@163.com

$\triangle$ Chang-Ming Huang hcmlr2002@163.com

Ping Li pingli811002@163.com

\section{Introduction}

Gastric cancer (GC) is the fifth-most common malignancy in humans and ranks third in tumor-related mortality [1]. Although scholars have worked hard to improve the diagnosis and treatment of GC, the recurrence rate of patients with GC is still high, and long-term survival is still not

1 Department of Gastric Surgery, Fujian Medical University Union Hospital, No. 29 Xinquan Road, Fuzhou 350001, Fujian, China

2 Key Laboratory of Ministry of Education of Gastrointestinal Cancer, Fujian Medical University, Fuzhou, Fujian, China 
optimistic. It is imperative to explore new promising prognostic indicators.

In 1863, Virchow first discovered the relationship between inflammation and cancer [2]. Subsequently, more and more scholars carried out related research. Many studies have shown that inflammatory indices, such as the neutrophil-lymphocyte ratio (NLR) and the systemic immuneinflammation index (SII), are independent prognostic factors for GC, and platelet-lymphocyte ratio (PLR) is closely related to postoperative peritoneal metastasis [3-5]. It is expected that the combination of TNM stage and inflammatory index could better predict the prognosis of patients with GC. Liu found that C-Reactive protein/Albumin (CRP/ ALB) was superior to traditional inflammatory indices in predicting long-term survival of GC [6]. Studies have shown that prealbumin can be used as a parameter of nutritional status evaluation [7] and is superior to albumin (ALB) [8]. What's more, prealbumin is also associated with postoperative recovery $[9,10]$ and is an independent predictor of prognosis in patients with malignancies [11-14]. However, the sample size of the previous studies was small, and they did not explore whether prealbumin was associated with postoperative recurrence after radical gastrectomy. Therefore, according to the above theoretical basis, we hypothesized that PALB could substitute for ALB to combine with CRP to construct a new inflammatory index: $\mathrm{CRP} /$ prealbumin ratio (CRP/PALB). In addition, the present study used prospective clinical trial data to investigate whether CRR/PALB could effectively predict postoperative recurrence of GC and compared the predictive value with traditional inflammatory indices.

\section{Materials and methods}

\section{Patients}

Between January 1, 2015 and April 1, 2016, a total of 438 patients admitted to Fujian Medical University Union Hospital were recruited to the trial, and 419 patients were included in the final analysis. (ClinicalTrials.gov number NCT02327481) [15]. The details about inclusion, exclusion, quality control and randomization have been previously reported $[15,16]$. The prospective, phase 3 randomized controlled trial (RCT) was conducted to determine whether the use of 3D laparoscopic gastrectomy would shorten the operation time compared with the use of the traditional 2D procedure. This RCT was conducted in accordance with the protocol that was approved by the institutional review boards of our hospital. All candidates were well informed and gave their full consent after they had received a verbal explanation of the study and an informational document $[15,16]$.
The present study is a sub-study of the RCT. After excluding ten patients with neuroendocrine carcinoma, six patients with palliative surgery and two patients without evidence for GC, the present analysis was restricted to 401 patients for whom curative gastrectomies were performed and for whom postoperative pathology confirmed stage I, II, or III gastric adenocarcinoma $\left(\mathrm{pT}_{1-4 \mathrm{a}} \mathrm{N}_{0-3} \mathrm{M}_{0}\right.$ ) according to the 7 th American Joint Committee on Cancer staging [17].

\section{Definitions}

Preoperative measurements of complete blood counts (CBC), CRP, ALB and PALB were derived within the 7 days prior to surgery. All the blood were recorded from the same sample.

Glasgow prognostic score (GPS): 0: CRP $\leq 10 \mathrm{mg} / \mathrm{L}$ and $\mathrm{ALB} \geq 35 \mathrm{~g} / \mathrm{L} ; 1: \mathrm{CRP}>10 \mathrm{mg} / \mathrm{L}$ or $\mathrm{ALB}<35 \mathrm{~g} / \mathrm{L} ; 2$ : $\mathrm{CRP}>10 \mathrm{mg} / \mathrm{L}$ and ALB $<35 \mathrm{~g} / \mathrm{L}$ [18].

Modified Glasgow prognostic score (mGPS): 0: $\mathrm{CRP} \leq 10 \mathrm{mg} / \mathrm{L}$ and any levels of ALB concentration; 1 : $\mathrm{CRP}>10 \mathrm{mg} / \mathrm{L}$ and normal levels of ALB ( $\geq 35 \mathrm{~g} / \mathrm{L}) ; 2$ : $\mathrm{CRP}>10 \mathrm{mg} / \mathrm{L}$ and ALB $<35 \mathrm{~g} / \mathrm{L}$ [19].

$N L R$ the ratio of the number of neutrophils to the number of lymphocytes.

$P L R$ the ratio of the number of platelets to the number of lymphocytes.

SII SII = platelets*NLR [4].

$C R P / A L B$ The CRP level divided by the ALB level.

$C R P / P A L B$ The CRP level divided by the PALB level.

Recurrence Recurrence was diagnosed with radiologic findings or biopsies of suspicious lesions. The recurrencefree survival period (RFS) was defined as the period from the date of surgery to the date of recurrence or last followup without recurrence. For RFS, patients who died without known tumor recurrence were censored at the last documented evaluation. Recurrence patterns were classified as local recurrence (LR) (anastomotic or gastric remnant), lymph node (LN) and distant metastasis (DM) (peritoneal, hepatic, pulmonary, or other sites of metastatic disease) $[20,21]$. Post-recurrence survival (PRS) was defined as the period from first recurrence to either death or the last follow-up.

\section{Follow-up}

The cutoff date for this analysis was April 2018, at which time all patients had reached a minimum of 24 months of follow-up, with a median follow-up of 24 (range 3-35) months since randomization. Postoperative follow-ups were performed every 3 months for 2 years, and then every 6 months from years 3 to 5. Most routine patient follow-up appointments included a physical examination, laboratory tests, chest radiography, 
abdominal ultrasonography or $\mathrm{CT}$ and an annual endoscopic examination.

\section{Statistical analysis}

Continuous variables are reported as means \pm SD. Categorical and continuous variables were compared using a $\chi^{2}$ test or Fisher's exact test and a t test, respectively. Receiver operating characteristic (ROC) curves and time-dependent ROC curves were generated, and the $\mathrm{C}$-index was calculated to evaluate the discriminative ability of the inflammatory indices. The optimal cut-off values of CRP/PALB and traditional inflammatory indices were obtained through ROC curve. By calculating the Youden index corresponding to different cut-off values of each inflammatory index in the ROC curve, and the corresponding cut-off values of the maximum value of Youden index were used to divide the patients into two groups [22]. The time-dependent ROC curve analysis is an extension of the ROC curve, which assesses the discriminatory power of continuous variables for time-dependent disease outcomes [23]. Differences between the C-index and areas under the curve (AUC) were compared. RFS was assessed using the Kaplan-Meier method. Cox proportional hazards regression model was used to identify the independent predictors associated with RFS. Variables with a value of $p<0.05$ in the univariate analysis were subsequently included in a multivariate analysis. Statistical analyses were performed using SPSS v.18.0 for Windows (SPSS Inc., Chicago, IL, USA) and R (https://www.r-project.org/). $p$ values less than 0.05 were considered statistically significant.

\section{Results}

\section{Clinicopathological characteristics}

The characteristics of the patients $(n=401)$ are listed in Table 1. The optimal cut-off point for preoperative CRP/ PALB for postoperative recurrence was obtained by ROC curve, which was 17.7, and the entire sample was divided into a low CRP/PALB $(\mathrm{LCP})$ group $(\mathrm{CRP} / \mathrm{PALB}<17.7)$ and a high CRP/PALB $(\mathrm{HCP})$ group (CRP/PALB $\geq 17.7)$ based on this cutoff point, including 245 and 156 patients, respectively. Compared with the LCP group, patients in the HCP group were older ( 60.4 vs $57.4, p=0.006)$ and were closely associated with poor clinical characteristics, including tumor size, $\mathrm{T}$ stage, $\mathrm{N}$ stage, TNM stage, and other inflammatory indices, all $p<0.05$.

\section{Comparing the recurrence prediction value of CRP/ PALB with traditional inflammatory indices}

To compare the predictive power of CRP/PALB with other inflammatory indices for postoperative recurrence, we
Table 1 Clinicopathological characteristics

\begin{tabular}{|c|c|c|c|}
\hline & $\begin{array}{l}\text { CRP/ } \\
\text { PALB }<17.7 \\
(n=245)\end{array}$ & $\begin{array}{l}\text { CRP/ } \\
\text { PALB } \geq 17.7 \\
(n=156)\end{array}$ & $p$ value \\
\hline Age (years) & $57.4 \pm 10.2$ & $60.4 \pm 10.1$ & 0.006 \\
\hline $\operatorname{Sex} n(\%)$ & & & 1.000 \\
\hline Male & $166(67.8 \%)$ & $105(67.3 \%)$ & \\
\hline Female & $79(32.2 \%)$ & $51(32.7 \%)$ & \\
\hline ASA $n(\%)$ & & & 1.000 \\
\hline$<3$ & $240(98.0 \%)$ & $153(98.0 \%)$ & \\
\hline$\geq 3$ & $5(2.0 \%)$ & $3(2.0 \%)$ & \\
\hline Tumor diameter (mm) & $36.3 \pm 21.1$ & $49.5 \pm 23.8$ & $<0.001$ \\
\hline Tumor location $n(\%)$ & & & 0.019 \\
\hline Upper & $61(24.9 \%)$ & $56(35.9 \%)$ & \\
\hline Middle & $50(20.4 \%)$ & $19(12.2 \%)$ & \\
\hline Lower & $121(49.4 \%)$ & $70(44.9 \%)$ & \\
\hline Mix & $10(4.1 \%)$ & $14(9.0 \%)$ & \\
\hline Gastrectomy extent $n(\%)$ & & & 0.010 \\
\hline Distal & $115(46.9 \%)$ & $53(34.0 \%)$ & \\
\hline Total & $130(53.1 \%)$ & $103(66.0 \%)$ & \\
\hline Reconstruction & & & 0.014 \\
\hline B-I & $62(25.3 \%)$ & $22(14.1 \%)$ & \\
\hline B-II & $53(21.6 \%)$ & $31(19.9 \%)$ & \\
\hline Roux-en-Y & $130(53.1 \%)$ & $103(66.0 \%)$ & \\
\hline Pathological type $n(\%)$ & & & 0.941 \\
\hline Differentiated & $103(42.0 \%)$ & $65(41.7 \%)$ & \\
\hline Undifferentiated & $142(58.0 \%)$ & $91(58.3 \%)$ & \\
\hline $\begin{array}{l}\text { Lymphovascular invasion } \\
n(\%)\end{array}$ & & & 0.001 \\
\hline No & $156(63.7 \%)$ & $72(46.2 \%)$ & \\
\hline Yes & $89(36.3 \%)$ & $84(53.8 \%)$ & \\
\hline GPS & & & $<0.001$ \\
\hline 0 & $225(91.8 \%)$ & $98(62.8 \%)$ & \\
\hline 1 & $20(8.2 \%)$ & $48(30.8 \%)$ & \\
\hline 2 & $0(0.0 \%)$ & $10(6.4 \%)$ & \\
\hline mGPS & & & $<0.001$ \\
\hline 0 & $245(100.0 \%)$ & $114(73.1 \%)$ & \\
\hline 1 & $0(0.0 \%)$ & $32(20.5 \%)$ & \\
\hline 2 & $0(0.0 \%)$ & $10(6.4 \%)$ & \\
\hline SII & & & $<0.001$ \\
\hline$<784.7$ & $199(81.2 \%)$ & $85(54.5 \%)$ & \\
\hline$\geq 784.7$ & $46(18.8 \%)$ & $71(45.5 \%)$ & \\
\hline NLR & & & $<0.001$ \\
\hline$<3.1$ & $204(83.3 \%)$ & $97(62.2 \%)$ & \\
\hline$\geq 3.1$ & $41(16.7 \%)$ & $59(37.8 \%)$ & \\
\hline PLR & & & $<0.001$ \\
\hline$<133.2$ & $125(51.0 \%)$ & $50(32.1 \%)$ & \\
\hline$\geq 133.2$ & $120(49.0 \%)$ & $72(67.9 \%)$ & \\
\hline CRP/ALB & & & $<0.001$ \\
\hline$<0.143$ & $245(100.0 \%)$ & $69(44.2 \%)$ & \\
\hline$\geq 0.143$ & $0(0.0 \%)$ & $87(55.8 \%)$ & \\
\hline pT stage $n(\%)$ & & & $<0.001$ \\
\hline
\end{tabular}


Table 1 (continued)

\begin{tabular}{|c|c|c|c|}
\hline & $\begin{array}{l}\text { CRP/ } \\
\text { PALB }<17.7 \\
(n=245)\end{array}$ & $\begin{array}{l}\text { CRP/ } \\
\text { PALB } \geq 17.7 \\
(n=156)\end{array}$ & $p$ value \\
\hline $\mathrm{T} 1$ & $98(40.0 \%)$ & $27(17.3 \%)$ & \\
\hline $\mathrm{T} 2$ & $31(12.7 \%)$ & $12(7.7 \%)$ & \\
\hline $\mathrm{T} 3$ & $72(29.4 \%)$ & $55(35.3 \%)$ & \\
\hline $\mathrm{T} 4$ & $44(18.0 \%)$ & $62(39.7 \%)$ & \\
\hline pN stage $n(\%)$ & & & $<0.001$ \\
\hline No & $117(47.8 \%)$ & $41(26.3 \%)$ & \\
\hline N1 & $42(17.1 \%)$ & $19(12.2 \%)$ & \\
\hline N2 & $38(15.5 \%)$ & $28(17.9 \%)$ & \\
\hline N3 & $48(19.6 \%)$ & $68(43.6 \%)$ & \\
\hline $\operatorname{pTNM} n(\%)$ & & & $<0.001$ \\
\hline I & $103(42.0 \%)$ & $32(20.5 \%)$ & \\
\hline II & $60(24.5 \%)$ & $24(15.4 \%)$ & \\
\hline III & $82(33.5 \%)$ & $100(64.1 \%)$ & \\
\hline Recurrence $n(\%)$ & & & $<0.001$ \\
\hline No & $223(91.0 \%)$ & $102(65.4 \%)$ & \\
\hline Yes & $22(9.0 \%)$ & $54(34.6 \%)$ & \\
\hline Death $n(\%)$ & & & $<0.001$ \\
\hline No & $232(94.7 \%)$ & $110(70.5 \%)$ & \\
\hline Yes & $13(5.3 \%)$ & $46(29.5 \%)$ & \\
\hline
\end{tabular}

established ROC curves for all inflammatory indices (Supplemental Fig. 1). By comparing the AUC and C-index of each inflammatory index, it was confirmed that CRP/ PALB had the highest AUC (0.707) and C-index (0.716), all $p<0.05$. In addition, we constructed the time-dependent ROC curves for both CRP/PALB and CRP/ALB and found that the AUC of CRP/PALB was persistently superior to CRP/ALB throughout the observation period (Table 2; Fig. 1).

\section{Comparison of recurrence patterns between 2 groups}

Figure 2 shows a comparison of postoperative recurrence patterns between the HCP and LCP groups. A total of 54 patients $(34.6 \%)$ and 22 patients $(9.0 \%)$ had postoperative recurrences in the HCP group and LCP group, respectively, $p<0.001$. Stratified analysis found that the incidence of DM, $\mathrm{LN}$, and LR was significantly higher in the HCP group than those in the LCP group (26.3\% vs $6.5 \%, 15.4 \%$ vs $2.4 \%$, $6.4 \%$ vs $1.6 \%$, respectively), all $p<0.05$.

\section{Comparison of recurrence time between 2 groups}

The average recurrence time was 10.1 months in the HCP group and 17.7 months in the LCP group, $p<0.001$. Stratified analysis found that the average recurrence time of DM and LR were significantly earlier in the HCP group than in

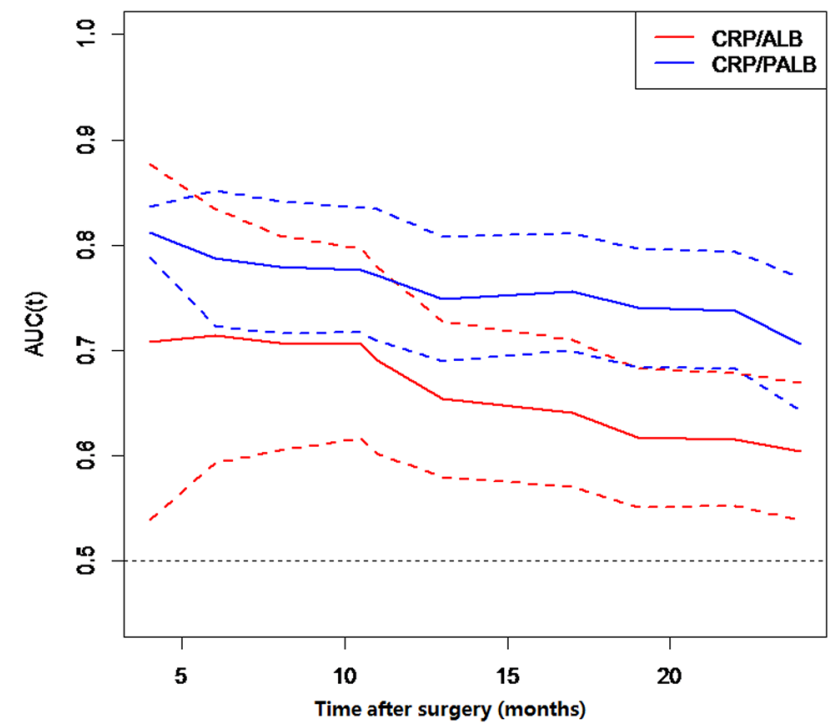

Fig. 1 Time-dependent receiver operating characteristic (ROC) curves for CRP/PALB and CRP/ALB

the LCP group (9.5 vs 17.2 months, $p<0.001 ; 11.3$ vs 23.0 months, $p=0.006$, respectively) (Supplementary Table 1 ).

\section{Univariate and multivariate analyses of factors associated with recurrence-free survival}

Univariate analysis of RFS in the whole sample revealed that tumor size, pTNM stage, differentiation type, lymphovascular invasion, reconstruction methods, GPS, mGPS, SII, NLR, CRP/ALB, CRP/PALB were related to RFS. Further multivariate analyses revealed that NLR, CRP/PALB, pTNM stage, and lymphovascular invasion were independent risk factors for postoperative recurrence (Table 3).

\section{Incorporation of preoperative CRP/prealbumin levels into conventional model}

According to the results of multivariate analysis, a new prognostic prediction system (model A) was established by combining preoperative CRP/PALB, NLR, pTNM stage and lymphovascular invasion. The results showed that the C-index of model A was significantly higher than that of the prognostic prediction system without CRP/PALB (model B). The C index was 0.846 (95\% CI 0.810-0.882) and 0.795 (95\% CI 0.756-0.833), respectively, $p<0.001$.

\section{Stratified analysis of recurrence-free survival and overall survival}

As Fig. 3a shows, the RFS of the HCP group was significantly lower than that of the LCP group (65.4\% vs $87.3 \%$, $p<0.001)$. According to the 7th AJCC-TNM staging system, 
Table 2 Comparison of the AUC and C-index between the inflammatory indices

\begin{tabular}{lllllll}
\hline Inflammatory indices & AUC & $95 \%$ CI & $p$ value* & C-index & $95 \%$ CI & $p$ value** \\
\hline GPS & 0.562 & $0.512-0.611$ & $<0.001$ & 0.57 & $0.516-0.623$ & $<0.001$ \\
mGPS & 0.558 & $0.508-0.608$ & $<0.001$ & 0.561 & $0.515-0.608$ & $<0.001$ \\
SII & 0.619 & $0.570-0.667$ & 0.032 & 0.645 & $0.581-0.708$ & 0.03 \\
NLR & 0.592 & $0.543-0.641$ & 0.007 & 0.604 & $0.539-0.670$ & 0.001 \\
PLR & 0.584 & $0.534-0.633$ & 0.004 & 0.596 & $0.532-0.660$ & $<0.001$ \\
CRP/ALB & 0.662 & $0.597-0.727$ & $<0.001$ & 0.672 & $0.615-0.728$ & 0.002 \\
CRP/PALB & 0.707 & $0.660-0.752$ & - & 0.716 & $0.664-0.767$ & - \\
\hline
\end{tabular}

*Comparison of AUC values between the CRP/PALB and other inflammatory indices

**Comparison of C-index between the CRP/PALB and other inflammatory indices

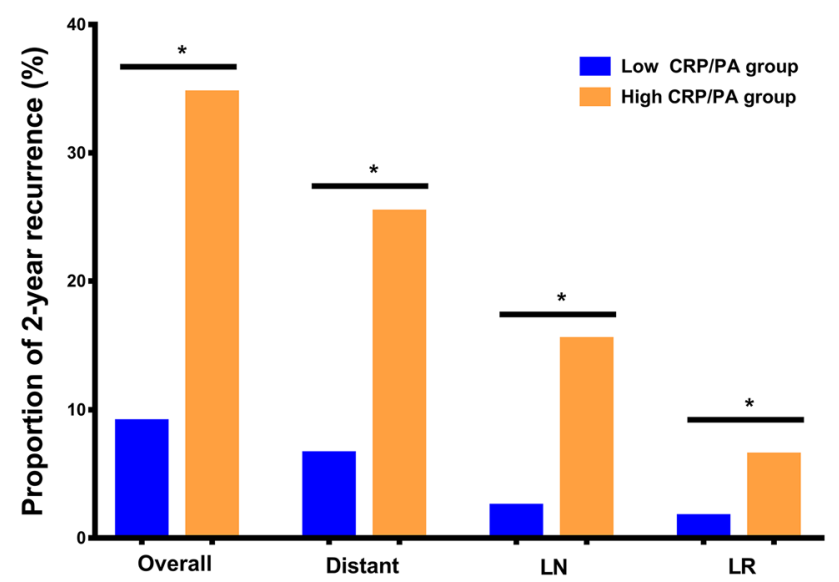

Fig. 2 Recurrence patterns according to histologic subtype between LCP and HCP groups. $L N$ lymph node; $L R$ locoregional, $* p<0.05$

the two groups of patients were stratified into pathological (p) stages I, II and III, as shown in Fig. 3b-d. Stratified analysis showed that there was no statistically significant difference in RFS between the two groups in patients with $p$ stage I and II, with $p$ values of 0.734 and 0.308 , respectively. In $p$ stage III patients, the RFS of the HCP group was significantly lower than that of the LCP group (49.0\% vs $71.8 \%$, $p<0.001)$. In addition, we compared the overall survival (OS) of the two groups. We found that OS was significantly lower in HCP group when compared with LCP group (69.6\% vs $94.7 \%, p<0.001)$. Stratified analysis showed that there was no statistically significant difference in OS between the two groups in patients with $\mathrm{p}$ stage I and II, with $p$ values of 0.701 and 0.315 , respectively. In p stage III patients, the OS of the HCP group was significantly lower than that of the LCP group (55.6\% vs $89.0 \%, p<0.001$ )(Supplemental Fig. 2A-D).

\section{Post-recurrence survival}

Among the 76 patients diagnosed with recurrence, the mean PRS was 7 months. Figure 4 a shows that the PRS of patients in the HCP group was significantly shorter than that of the LCP group, $p=0.010$. Since the number of recurrences in the patients with $\mathrm{p} \mathrm{I}$ and II stages were small, with only 3 and 4 cases, respectively, patients with recurrence were divided into $\mathrm{p} \mathrm{I-II}$ and $\mathrm{p} \mathrm{III} \mathrm{stages.} \mathrm{Survival} \mathrm{curve} \mathrm{analysis}$ in $\mathrm{p} \mathrm{I-II} \mathrm{patients} \mathrm{revealed} \mathrm{a} \mathrm{similar} \mathrm{PRS} \mathrm{between} \mathrm{the} \mathrm{HCP}$ group and the LCP group (Fig. $4 b$ ), $p=0.304$. In the $p$ III stage, PRS was significantly shorter in the HCP group than the LCP group (Fig. 4c), $p=0.015$. In addition, patients with recurrence were further stratified into three groups: DM, LN and LR. Stratified analysis showed that in the HCP group with DM or LR, the PRS was significantly shorter than the LCP group, and $p$ values were 0.018 and 0.023 , respectively. For patients with $\mathrm{LN}$, no significant difference was observed in PRS between the HCP group and the LCP group, $p=0.329$. (Fig. 4d-f).

\section{Discussion}

Since Virchow first discovered the relationship between inflammation and cancer [2], more and more evidence showed that tumor progression was not only related to the intrinsic properties of tumor cells but also inseparable from the body's inflammatory immune response [24]. The characteristics of cancer-associated inflammation include the infiltration of inflammatory cells and the production of inflammatory factors in tumor tissues, tissue remodeling, tissue repair, and angiogenesis [25]. Inflammatory indices derived from the CBC, such as NLR, PLR, and SII, were considered to be associated with a tumor-promoting role (neutrophil, platelet) or an anti-tumor system (lymphocyte) $[26,27]$. These theories led to an in-depth study of these inflammatory indices recently, confirming that inflammatory index was closely related to the prognosis of many tumors [26, 28-31].

CRP is mainly produced by liver cells. Its rapid increase in serum concentration is related to tumor necrosis factor $\alpha$ (TNF- $\alpha$ ), interleukin 6 (IL-6) and other proinflammatory cytokines [32]. These proinflammatory cytokines accelerate 
Table 3 Univariate and multivariate analyses of factors associated with recurrence-free survival

\begin{tabular}{|c|c|c|c|c|c|c|}
\hline & \multicolumn{3}{|c|}{ Univariate analysis } & \multicolumn{3}{|c|}{ Multivariate analysis } \\
\hline & $\mathrm{HR}$ & $95 \% \mathrm{CI}$ & $p$ value & HR & $95 \% \mathrm{CI}$ & $p$ value \\
\hline \multicolumn{7}{|l|}{ Sex } \\
\hline Female & 1.000 & & & & & \\
\hline Male & 1.080 & $0.646-1.803$ & 0.770 & & & \\
\hline \multicolumn{7}{|l|}{ Age (years) } \\
\hline$<65$ & 1.000 & & & & & \\
\hline$\geq 65$ & 0.975 & $0.584-1.629$ & 0.923 & & & \\
\hline \multicolumn{7}{|l|}{ ASA } \\
\hline$<3$ & 1.000 & & & & & \\
\hline$\geq 3$ & 0.774 & $0.107-5.578$ & 0.799 & & & \\
\hline \multicolumn{7}{|c|}{ Tumor diameter (mm) } \\
\hline$<50$ & 1.000 & & & 1.000 & & \\
\hline$\geq 50$ & 3.572 & $2.213-5.765$ & $<0.001$ & 1.320 & $0.829-2.102$ & 0.242 \\
\hline \multicolumn{7}{|l|}{ Tumor location } \\
\hline Upper & 1.000 & & & & & \\
\hline Middle & 0.515 & $0.243-1.092$ & 0.084 & & & \\
\hline Lower & 0.602 & $0.358-1.012$ & 0.055 & & & \\
\hline Mix & 1.105 & $0.537-2.275$ & 0.786 & & & \\
\hline \multicolumn{7}{|l|}{ Gastrectomy extent } \\
\hline Distal & 1.000 & & & & & \\
\hline Total & 1.506 & $0.913-2.485$ & 0.109 & & & \\
\hline \multicolumn{7}{|l|}{ Reconstruction } \\
\hline B-I & 1.000 & & & 1.000 & & \\
\hline B-II & 3.375 & $1.339-8.506$ & 0.010 & 1.522 & $0.441-5.263$ & 0.507 \\
\hline Roux-en-Y & 3.554 & $1.526-8.277$ & 0.003 & 1.101 & $0.204-5.951$ & 0.911 \\
\hline \multicolumn{7}{|l|}{ Pathological type } \\
\hline Differentiated & 1.000 & & & 1.000 & & \\
\hline Undifferentiated & 2.427 & $1.430-4.118$ & 0.001 & 1.621 & $0.972-2.703$ & 0.064 \\
\hline \multicolumn{7}{|c|}{ Lymphovascular invasion } \\
\hline No & 1.000 & & & 1.000 & & \\
\hline Yes & 7.433 & $4.089-13.512$ & $<0.001$ & 2.906 & $1.499-5.633$ & 0.002 \\
\hline \multicolumn{7}{|l|}{ GPS } \\
\hline 0 & 1.000 & & & 1.000 & & \\
\hline 1 & 1.660 & $0.962-2.864$ & 0.068 & 1.163 & $0.719-1.881$ & 0.538 \\
\hline 2 & 4.246 & $1.696-10.633$ & 0.002 & 2.174 & $0.528-8.947$ & 0.282 \\
\hline \multicolumn{7}{|l|}{ mGPS } \\
\hline 0 & 1.000 & & & 1.000 & & \\
\hline 1 & 2.008 & $1.029-3.920$ & 0.041 & 1.113 & $0.909-1.362$ & 0.299 \\
\hline 2 & 4.132 & $1.658-10.302$ & 0.002 & 2.174 & $0.528-8.947$ & 0.282 \\
\hline \multicolumn{7}{|l|}{ SII } \\
\hline$<784.7$ & 1.000 & & & 1.000 & & \\
\hline$\geq 784.7$ & 2.844 & $1.813-4.460$ & $<0.001$ & 1.303 & $0.731-2.324$ & 0.370 \\
\hline \multicolumn{7}{|l|}{ NLR } \\
\hline$<3.1$ & 1.000 & & & 1.000 & & \\
\hline$\geq 3.1$ & 2.404 & $1.516-3.814$ & $<0.001$ & 1.853 & $1.162-2.956$ & 0.010 \\
\hline \multicolumn{7}{|l|}{ PLR } \\
\hline$<133.2$ & 1.000 & & & & & \\
\hline$\geq 133.2$ & 1.558 & $0.969-2.504$ & 0.067 & & & \\
\hline \multicolumn{7}{|l|}{ CRP/ALB } \\
\hline$<0.143$ & 1.000 & & & 1.000 & & \\
\hline
\end{tabular}


Table 3 (continued)

\begin{tabular}{|c|c|c|c|c|c|c|}
\hline & \multicolumn{3}{|c|}{ Univariate analysis } & \multicolumn{3}{|c|}{ Multivariate analysis } \\
\hline & HR & $95 \% \mathrm{CI}$ & $p$ value & HR & $95 \% \mathrm{CI}$ & $p$ value \\
\hline$\geq 0.143$ & 2.601 & $1.637-4 . .134$ & $<0.001$ & 1.495 & $0.440-5.075$ & 0.519 \\
\hline \multicolumn{7}{|c|}{ CRP/PALB } \\
\hline$<17.7$ & 1.000 & & & 1.000 & & \\
\hline$\geq 17.7$ & 4.673 & $2.844-7.678$ & $<0.001$ & 2.760 & $1.667-4.569$ & $<0.001$ \\
\hline \multicolumn{7}{|l|}{ pTNM } \\
\hline I & 1.000 & & & 1.000 & & \\
\hline II & 2.226 & $0.498-9.949$ & 0.295 & 1.136 & $0.240-5.371$ & 0.872 \\
\hline III & 20.751 & $6.530-65.944$ & $<0.001$ & 7.136 & $1.989-25.597$ & 0.003 \\
\hline
\end{tabular}
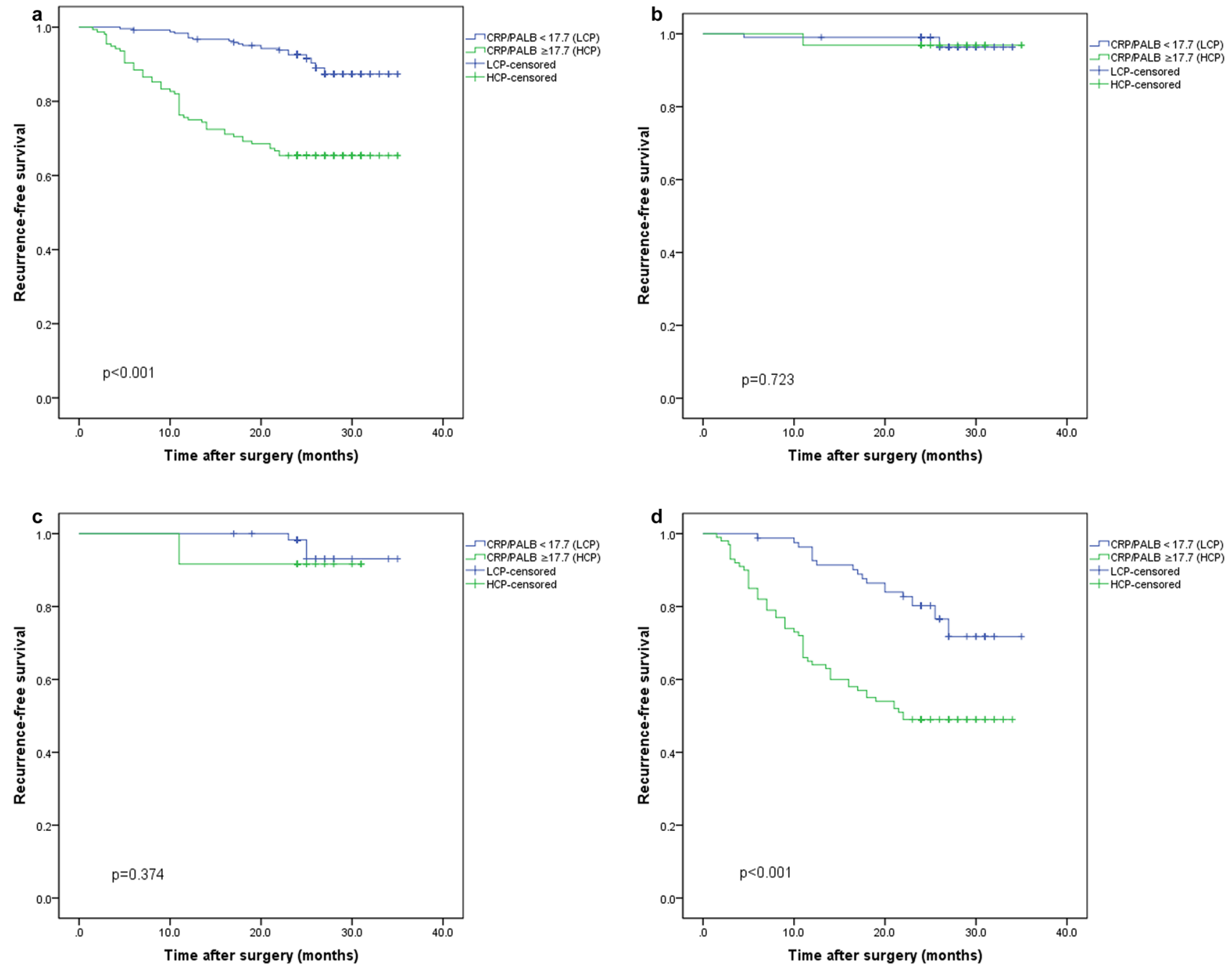

Fig. 3 Comparison of recurrence-free survival between the HCP and LCP groups according to pathological (p) stage. a All patients. b p stage (I) c p stage (II) d p stage III

angiogenesis, which in turn enhances the progression and metastasis of malignant tumors [33, 34]. ALB is closely related to nutritional status, which is a good indicator of immune status. In addition, malnutrition is closely related to the decrease of immune function, which weakens the body's anti-tumor immunity. Based on the above theory, a series of studies have confirmed that CRP and ALB are significantly associated with the prognosis of various tumors [35-37], 

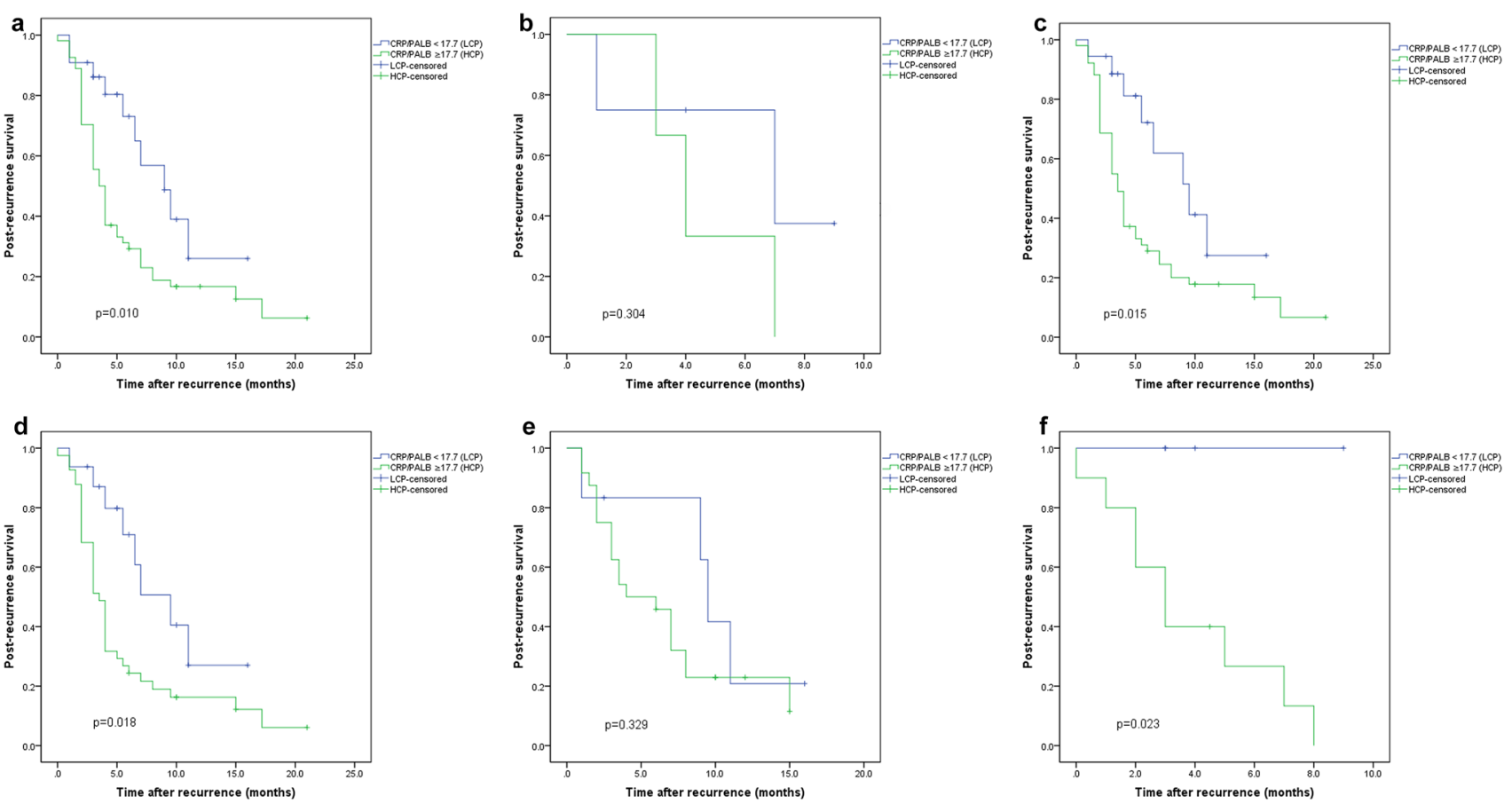

Fig. 4 Comparison of post-recurrence survival in patients with recurrence between the HCP and LCP groups according to pathological (p) stage and recurrences patterns. a All patients with recurrence. $\mathbf{b} \mathrm{p}$ stage I-II. c p stage III. d Patients with distant metastasis. e Patients with lymph nodes metastasis. f Patients with local recurrence

to analyze patients with early colon cancer and found that NLR is closely related to postoperative recurrence [45]. A retrospective study conducted by Mohammad found that SII is an independent risk factor for postoperative recurrence in patients with pancreatic cancer [46]. Kentaro reported that preoperative ALB is closely associated with postoperative recurrence of NSCLC [37]. In GC, Powell and mohri respectively confirmed that mGPS and GPS were related to recurrence $[47,48]$. This study, for the first time, innovatively used PALB in place of ALB, combined with CRP, to construct a new inflammatory index: CRP/PALB. We also aimed to explore if preoperative elevated CRP/PALB was related to postoperative recurrence in GC patients. Our current study found a strong correlation between $\mathrm{HCP}$ and many poor clinical features. In addition, multivariate analysis showed that CRP/PALB, not CRP/ALB, was associated with RFS. CRP/PALB also had the highest AUC and C-index when compared with the other inflammatory indices, including CRP/ALB. What's more, model including CRP/PALB is superior than that constructed without CRP/PALB in predicting recurrence after radical gastrectomy.

For patients with recurrence after radical gastrectomy, long-term survival is not optimistic. However, few studies related to PRS have been reported. Lee conducted a followup of 386 patients with postoperative recurrence of GC, revealing a median survival of 8.4 months after recurrence and a close correlation between the Lauren classification tion from scholars. Gennaro used propensity score matching 
and PRS [49]. The current study demonstrated that preoperative HCP is associated with DM, LN and LR. Moreover, preoperative HCP significantly reduced the PRS, especially in p stage III patients and in patients with DM and LR. Therefore, based on the findings of this study, we believe that patients with preoperative CRP/PALB $\geq 17.7$ should be followed-up closely, especially in $p$ stage III patients. Additionally, patients with HCP should be given aggressive treatment after recurrence to improve PRS, especially in $\mathrm{p}$ stage III patients and in patients with DM or LR.

Several limitations remain in our present study. First, this is a single-center study with a small sample size. Second, it lacks basic research to explore the detailed mechanism for how CRP/PALB is involved in the recurrence of GC. Third, we did not analyze the impact of chemotherapy on recurrence patterns. Forth, this is an exploratory analysis and a multicenter validation study is warranted. Nevertheless, the current study found that preoperative CRP/PALB is superior to the other inflammatory indices and closely related to RFS. Though the follow-up time is short for our cohort, most of the patients with GC will occur recurrence within 2 years after radical gastrectomy, which makes our research still have certain clinical significance.

\section{Conclusion}

The prediction value of preoperative CRP/PALB for the recurrence of GC after radical resection is significantly better than other traditional inflammatory indices. Patients with HCP are more likely to have DM, LN and LR than patients with LCP. HCP significantly reduces the PRS of patients with p stage III or patients with DM or LR. These patients are more likely to benefit from a rigorous follow-up strategy.

Acknowledgements This study was funded by the Scientific and technological innovation joint capital projects of Fujian Province (2016Y9031). Construction Project of Fujian Province Minimally Invasive Medical Center (No. [2017]171). The second batch of special support funds for Fujian Province innovation and entrepreneurship talents (2016B013). QIHANG funds of Fujian Medical University (No. 2016QH025). Fujian province medical innovation project (2015-CXB16). Fujian provincial health and family planning commission joint project (WKJ2016-2-27). Chinese physicians association young physician respiratory research fund.

Author contributions JL, BX, ZZ and $\mathrm{CH}$ conceived the study, analyzed the data, and drafted the manuscript, C-HZ helped critically revise the manuscript for important intellectual content. PL, JX, JBW, J-XL, Q-YC, L-LC, ML, R-HT and Z-NH helped collect data and design the study.

\section{Compliance with ethical standards}

Conflict of interest There are no conflicts of interest or financial ties to disclose from any of author.
Human rights statement All procedures followed were in accordance with the ethical standards of the responsible committee on human experimentation (institutional and national) and with the Helsinki Declaration of 1964 and later versions.

Informed consent Informed consent or substitute for it was obtained from all patients for being included in the study.

\section{References}

1. Fock KM. Review article: the epidemiology and prevention of gastric cancer. Aliment Pharmacol Ther. 2014;40(3):250-60.

2. Balkwill F, Mantovani A. Inflammation and cancer: back to Virchow? Lancet. 2001;357(9255):539-45.

3. Miyamoto R, Inagawa $\mathrm{S}$, Sano $\mathrm{N}$, et al. The neutrophil-to-lymphocyte ratio (NLR) predicts short-term and long-term outcomes in gastric cancer patients. Eur J Surg Oncol. 2018;44(5):607-12.

4. Wang K, Diao F, Ye Z, et al. Prognostic value of systemic immune-inflammation index in patients with gastric cancer. Chin J Cancer. 2017;36(1):75.

5. Chen XD, Mao CC, Wu RS, et al. Use of the combination of the preoperative platelet-to-lymphocyte ratio and tumor characteristics to predict peritoneal metastasis in patients with gastric cancer. PLoS One. 2017;12(4):e0175074.

6. Liu X, Sun X, Liu J, et al. Preoperative C-reactive protein/albumin ratio predicts prognosis of patients after curative resection for gastric cancer. Transl Oncol. 2015;8(4):339-45.

7. Gonda K, Shibata M, Sato Y, et al. Elevated neutrophil-to-lymphocyte ratio is associated with nutritional impairment, immune suppression, resistance to S-1 plus cisplatin, and poor prognosis in patients with stage IV gastric cancer. Mol Clin Oncol. 2017;7(6):1073-8.

8. Unal D, Orhan O, Eroglu C, et al. Prealbumin is a more sensitive marker than albumin to assess the nutritional status in patients undergoing radiotherapy for head and neck cancer. Contemp Oncol (Pozn). 2013;17(3):276-80.

9. Wang J, Zhao J, Zhang Y, et al. Early enteral nutrition and total parenteral nutrition on the nutritional status and blood glucose in patients with gastric cancer complicated with diabetes mellitus after radical gastrectomy. Exp Ther Med. 2018;16(1):321-7.

10. Nikniaz Z, Somi MH, Nagashi S, et al. Impact of early enteral nutrition on nutritional and immunological outcomes of gastric cancer patients undergoing gastrostomy: a systematic review and meta-analysis. Nutr Cancer. 2017;69(5):693-701.

11. Shimura T, Shibata M, Gonda K, et al. Serum transthyretin level is associated with prognosis of patients with gastric cancer. J Surg Res. 2018;227:145-50.

12. Zhou H, Jiang X, Li Q, et al. A simple and effective prognostic staging system based on clinicopathologic features of intrahepatic cholangiocarcinoma. Am J Cancer Res. 2015;5(5):1831-43.

13. Kawai H, Ota $\mathrm{H}$. Low perioperative serum prealbumin predicts early recurrence after curative pulmonary resection for non-smallcell lung cancer. World J Surg. 2012;36(12):2853-7.

14. Han WX, Chen ZM, Wei ZJ, et al. Preoperative pre-albumin predicts prognosis of patients after gastrectomy for adenocarcinoma of esophagogastric junction. World J Surg Oncol. 2016;14(1):279.

15. Zheng $\mathrm{CH}, \mathrm{Lu}$ J, Zheng HL, et al. Comparison of 3D laparoscopic gastrectomy with a 2D procedure for gastric cancer: a phase 3 randomized controlled trial. Surgery. 2018;163(2):300-4.

16. Lu J, Zheng $\mathrm{CH}$, Zheng HL, et al. Randomized, controlled trial comparing clinical outcomes of $3 \mathrm{D}$ and $2 \mathrm{D}$ laparoscopic surgery for gastric cancer: an interim report. Surg Endosc. 2017;31(7):2939-45. 
17. Washington K. 7th edition of the AJCC cancer staging manual: stomach. Ann Surg Oncol. 2010;17(12):3077-9.

18. McMillan DC. The systemic inflammation-based Glasgow Prognostic Score: a decade of experience in patients with cancer. Cancer Treat Rev. 2013;39(5):534-40.

19. Park JH, Watt DG, Roxburgh CS, et al. Colorectal cancer, systemic inflammation, and outcome: staging the tumor and staging the host. Ann Surg. 2016;263(2):326-36.

20. Lee D, Son SY, Kim YB, et al. Neural invasion is a significant contributor to peritoneal recurrence in signet ring cell gastric carcinoma. Ann Surg Oncol. 2018;25:1167-1175

21. Jin LX, Moses LE, Squires MH III. Factors associated with recurrence and survival in lymph node-negative gastric adenocarcinoma: A 7-institution study of the US Gastric Cancer Collaborative. Ann Surg. 2015;262(6):999-1005. rd, et al.

22. Bantis LE, Nakas CT, Reiser B. Construction of confidence regions in the ROC space after the estimation of the optimal Youden index-based cut-off point. Biometrics. 2014;70(1):212-23.

23. Heagerty P, Lumley T, Pepe M. Time-dependent ROC curves for censored survival data and a diagnostic marker. Biometrics. 2000;56(2):337-44.

24. Alifano M, Mansuet-Lupo A, Lococo F, et al. Systemic inflammation, nutritional status and tumor immune microenvironment determine outcome of resected non-small cell lung cancer. PLoS One. 2014;9(9):e106914.

25. Mantovani A, Allavena P, Sica A, et al. Cancer-related inflammation. Nature. 2008;454(7203):436-44.

26. Fankhauser CD, Sander S, Roth L, et al. Systemic inflammatory markers have independent prognostic value in patients with metastatic testicular germ cell tumours undergoing first-line chemotherapy. Br J Cancer. 2018;118(6):825-30.

27. Thibodeau J, Bourgeois-Daigneault MC, Lapointe R. Targeting the MHC Class II antigen presentation pathway in cancer immunotherapy. Oncoimmunology. 2012;1(6):908-16.

28. Liu Z, Jin K, Guo M, et al. Prognostic value of the CRP/Alb ratio, a novel inflammation-based score in pancreatic cancer. Ann Surg Oncol. 2017;24(2):561-8.

29. Mano Y, Shirabe K, Yamashita Y, et al. Preoperative neutrophilto-lymphocyte ratio is a predictor of survival after hepatectomy for hepatocellular carcinoma: a retrospective analysis. Ann Surg. 2013;258(2):301-5.

30. Chovanec M, Cierna Z, Miskovska V, et al. Systemic immuneinflammation index in germ-cell tumours. Br J Cancer. 2018;118(6):831-8.

31. Shimada H, Takiguchi N, Kainuma O, et al. High preoperative neutrophil-lymphocyte ratio predicts poor survival in patients with gastric cancer. Gastric Cancer. 2010;13(3):170-6.

32. Gabay C, Kushner I. Acute-phase proteins and other systemic responses to inflammation. N Engl J Med. 1999;340(6):448-54.

33. Ilhan N, Ilhan N, Ilhan Y, et al. C-reactive protein, procalcitonin, interleukin-6, vascular endothelial growth factor and oxidative metabolites in diagnosis of infection and staging in patients with gastric cancer. World J Gastroenterol. 2004;10(8):1115-20.

34. Sansone P, Storci G, Tavolari S, et al. IL-6 triggers malignant features in mammospheres from human ductal breast carcinoma and normal mammary gland. J Clin Invest. 2007;117(12):3988-4002.

35. Grimm T, Buchner A, Schneevoigt B, et al. Impact of preoperative hemoglobin and CRP levels on cancer-specific survival in patients undergoing radical cystectomy for transitional cell carcinoma of the bladder: results of a single-center study. World J Urol. 2016;34(5):703-8.

36. Basu S, Harris H, Larsson A, et al. Is there any role for serum cathepsin S and CRP levels on prognostic information in breast cancer? The Swedish Mammography Cohort. Antioxid Redox Signal. 2015;23(16):1298-302.

37. Miura K, Hamanaka K, Koizumi T, et al. Clinical significance of preoperative serum albumin level for prognosis in surgically resected patients with non-small cell lung cancer: Comparative study of normal lung, emphysema, and pulmonary fibrosis. Lung Cancer. 2017;111:88-95.

38. Ishino Y, Saigusa S, Ohi M, et al. Preoperative C-reactive protein and operative blood loss predict poor prognosis in patients with gastric cancer after laparoscopy-assisted gastrectomy. Asian J Endosc Surg. 2014;7(4):287-94.

39. Takamori S, Toyokawa G, Shimokawa M, et al. The C-reactive protein/albumin ratio is a novel significant prognostic factor in patients with malignant pleural mesothelioma: a retrospective multi-institutional study. Ann Surg Oncol. 2018;25(6):1555-63.

40. Saito H, Kono Y, Murakami Y, et al. Prognostic significance of the preoperative ratio of $\mathrm{C}$-reactive protein to albumin and neutrophil-lymphocyte ratio in gastric cancer patients. World J Surg. 2018;42(6):1819-25.

41. Banks R, Forbes M, Storr M, et al. The acute phase protein response in patients receiving subcutaneous IL-6. Clin Exp Immunol. 1995;102(1):217-23.

42. Chertow GM, Ackert K, Lew NL, et al. Prealbumin is as important as albumin in the nutritional assessment of hemodialysis patients. Kidney Int. 2000;58(6):2512-7.

43. Myron Johnson A, Merlini G, Sheldon J, et al. Clinical indications for plasma protein assays: transthyretin (prealbumin) in inflammation and malnutrition. Clin Chem Lab Med. 2007;45(3):419-26.

44. Zhu HF, Li J, Huang L, et al. Intrahepatic cholangiocarcinoma: a clinicopathologic study of 37 resected cases. Hepatogastroenterology. 2013;60(122):263-7.

45. Galizia G, Lieto E, Zamboli A, et al. Neutrophil to lymphocyte ratio is a strong predictor of tumor recurrence in early colon cancers: a propensity score-matched analysis. Surgery. 2015;158(1):112-20.

46. Aziz MH, Sideras K, Aziz NA, et al. The systemic-immuneinflammation index independently predicts survival and recurrence in resectable pancreatic cancer and its prognostic value depends on bilirubin levels: a retrospective multicenter cohort study. Ann Surg. 2018. https://doi.org/10.1097/SLA.0000000000 002660

47. Powell A, Parkinson D, Patel N, et al. Prognostic significance of serum inflammatory markers in gastric cancer. J Gastrointest Surg. 2018;22(4):595-605.

48. Mohri Y, Tanaka K, Ohi M, et al. Inflammation-based prognostic score as a predictor of postoperative gastric cancer recurrence. Anticancer Res. 2012;32(10):4581-4.

49. Lee JH, Chang KK, Yoon C, et al. Lauren histologic type is the most important factor associated with pattern of recurrence following resection of gastric adenocarcinoma. Ann Surg. 2018;267(1):105-13. 